\title{
Frontier science
}

In the current discussions on the evolution of the International Agricultural Research System, for more efficiency with regard to the developing world, after the food crisis and financial crisis which have been so damaging, a key word which is strongly emerging is "Frontier science".

Science is more than ever considered as essential for addressing major challenges such as poverty alleviation, food and nutritional security and environmental issues, but it needs to be embedded in multidisciplinary and multisectorial approaches, in order to correctly address all these complex problems which, by nature, do not know disciplinary or sectorial frontiers.

Fruits are a good example of that.

The promotion of fruits and vegetables for health, which is seen as a more relevant way of addressing nutritional problems such as nutrient deficiencies and chronic diseases, must be supported by innovation calling for crop scientists, nutritionists, economists, sociologists, anthropologists, epidemiologists, medical science specialists, etc., also calling for strong interactions between agriculture, health, education, environment ...

It is urgent that research be implemented, recognized and evaluated with such a course of action.

Unfortunately, the current situation is quite different as researchers are commonly evaluated through their publications in peer-reviewed journals, most of them being mono-disciplinary journals dedicated to specific sectors, i.e., agronomic, medical, economic, social, human, ... sectors.

Therefore, there are very few places for multidisciplinary and/or multisectorial scientific papers!

Are scientific evaluation and publications thus limiting factors for correctly addressing the key challenges of our planet?

Frontier research is considered as essential but it will be ineffective if solutions are not found to attract researchers towards such approaches and, in particular, through relevant evaluation systems and publication support.

As an impact-driven scientific journal, Fruits has always been supportive of such approaches. More than ever it is ready to be active in Frontier Science and to host multidisciplinary papers from horticultural, health, education, and other sectors all together, where fruits will be a common denominator, within its direct mission to resolve problems with science, particularly in developing countries.

Dr. Jacky Ganry

Chairman of the Section on Tropical and Subtropical Fruits

ISHS 


\section{Une Science sans frontière}

Lors des discussions actuelles sur l'évolution du système international de recherche agricole pour plus d'efficacité vers le tiers monde, après les crises alimentaire et économique qui ont été si préjudiciables, un mot clé émerge fortement : "Science sans frontière ".

La Science est plus que jamais considérée comme essentielle pour relever d'importants défis tels que l'éradication de la pauvreté, la sécurité nutritionnelle et alimentaire, les questions environnementales, mais elle doit être englobée dans des approches multidisciplinaires et multisectorielles pour aborder correctement tous ces problèmes complexes qui, par nature, ne connaissent pas de frontières disciplinaires ou sectorielles.

Les fruits sont un bon exemple de tout cela.

La promotion des fruits et légumes pour la santé, qui est considérée comme une des voies les mieux appropriée pour aborder les problèmes de nutrition tels que les carences alimentaires et les maladies chroniques, doit être soutenue par l'innovation qui ellemême interpelle les agronomes, les nutritionnistes, les économistes, les sociologues, les anthropologues, les épidémiologistes, les spécialistes des sciences médicales, ..., et qui nécessite de fortes interactions entre agriculture, santé, éducation, environnement...

Il est urgent que la recherche soit mise en application, reconnue et évaluée avec une telle ligne de conduite.

Malheureusement, la situation actuelle est très différente car les chercheurs sont généralement évalués sur leurs publications dans des revues à comité de lecture, la plupart d'entre elles étant des journaux monodisciplinaires consacrés à des secteurs spécifiques, comme les secteurs agronomiques, médicaux, économiques, sociaux, humains, ... Par conséquent, il y a très peu de place pour des publications scientifiques pluridisciplinaires et/ou plurisectorielles!

L'évaluation et les publications scientifiques seraientelles ainsi des facteurs limitants pour relever correctement les grands défis de notre planète?

Une recherche sans frontière est considérée comme essentielle mais elle sera sans effet si des solutions ne sont pas trouvées pour attirer les chercheurs vers de telles approches et, en particulier, par l'utilisation de systèmes d'évaluation et de supports de publication appropriés.

En tant que périodique scientifique préoccupé par son impact sur le développement, Fruits a toujours été un supporter de telles approches. Plus que jamais, la revue est prête à ouvrer pour une science sans frontière et pour accueillir des articles pluridisciplinaires sur les secteurs de l'horticulture, de la santé, de l'éducation, et autres, tous confondus, pour lesquels les fruits seraient un dénominateur commun, et ce dans la droite ligne de sa mission de résolution des problèmes par la science, en particulier dans les pays en voie de développement.

Dr. Jacky Ganry Président de la section Fruits tropicaux et subtropicaux ISHS 